\title{
Organization and management system based on workflow and web technology
}

\author{
Xueqin Liu1, a , Huarui $\mathrm{Wu}^{1,2, \mathrm{~b}}$ \\ ${ }^{1}$ Beijing Research Center of Intelligent Equipment for Agriculture,Beijing \\ 100097,China \\ ${ }^{2}$ National Research Center of Intelligent Equipment for Agriculture, \\ Beijing 100097, China \\ aemail: liuxq@nercita.org.cn , bemail:wuhr@nercita.org.cn
}

\begin{abstract}
Workflow technology[1] is a business process modeling, process management and integration, and ultimately implement business process executing automatically. It is crucial to improve business process efficiency. With the popularity of the Internet and Web technologies, the traditional c/s mode is designed to automate the management system which can't satisfy the needs of users, so using b/s structure has become a major feature of the new generation of workflow management systems. The paper studies the workflow technology and web technology's application in Organization and management systems of sustainable urban development.
\end{abstract}

Keyword: workflow; b/s structure; struts; JSP; hibernate

\section{Introduction}

With the social production automation and process-oriented, workflow which bases on web plays more and more important role. According to Workflow Management Coalition definition[2][3],workflow is automatically execute business process function part or whole, performance that participants make files , information or task accordance with rules and make them passed between participants. This paper describes the design and implementation process organization and management system, process management module in the workflow. 


\section{Overview of workflow technology}

The concept of workflow generated from traditional manufacturing production organization. "Flow" reflects a change and the process of change, is an abstract concept. For the daily work of a series of relatively fixed order of business processes, resolve the process into multiple tasks and roles, according to certain rules to execute and monitor any sequence, into order to reach the purposes which improve efficiency, reduce costs and improve management level.

Workflow which is business processes partly or wholly automatically execute in the computer environment[4], is a series of tasks which interrelated and automatically running. A workflow includes a set of tasks' start and termination conditions, and a description of each task (or activities). Activities correspond to the business process tasks, primarily reflecting the business process to perform an action or operation, the connection between activities is on behalf of the rules and business process management process.

\section{WEB-based workflow development proposed and its advantages}

The success and popularity of web and the emergence of a variety of related technologies, many applications are ported to the web platform. Since 1995, the products of workflow suppliers and related research began transferred workflow management system to the web platform. Compared to other workflow systems ( such as :file-based, message-based workflow system, etc), the web-based workflow management system has many advantages, such as the future of the computer model, browser is desktop, clients are all web-enabled. The client of web-based workflow system only needs a browser, the browser via HTTP protocol to download the client program from web server to local machine executing, which can greatly simplify maintenance of the system. Web technology is currently one of the hottest technologies, new technologies emerge in endlessly, some original technologies are also integrated into the web technology. 


\section{The design and implementation of system framework}

With the rapid development of computer technology, information management has penetrated into all walks of life, in order to solve low organizational efficiency and running is not standardized problems of experimental Zone construction and management center, accelerate its process of information has become an inevitable trend. After Java matured into a cross-platform development language, develop cross-platform software system the language of Java is preferred, then use struts framework effectively separate JSP and java code, make develop more easily, and the paper applies workflow technology into web system[5], these technologies' application is effective to solve low organizational efficiency and process is not standardized problems of our sustainable development experimental zone and management center.

National sustainable development organization and management system includes four function roles, such are 21 Century Management Center(Role1), the experimental area (Role2), ministries (Role3) and expert (Role4). Each role's functional responsibilities show in figure (a), In the system module role1's functional responsibilities such as management role2, role3 and role4; Depending on the status of the workflow selection role4, and then according to role4's comments and scoring table audits the material submitted by role2 .Functional responsibilities of role2 are submitted material and view the material according to the workflow. Functional responsibilities of role3 are view material submitted by role2 and submits material to role1 based on workflow. Role4 can be subdivided into ordinary expert(role4.1 ) and ministries expert(role 4.2),role4.1 has the functions such as fill submissions, field trip role2 and fill inspect report according to inspect results; Role4.2 has the function responsibilities to fill scoring table. 


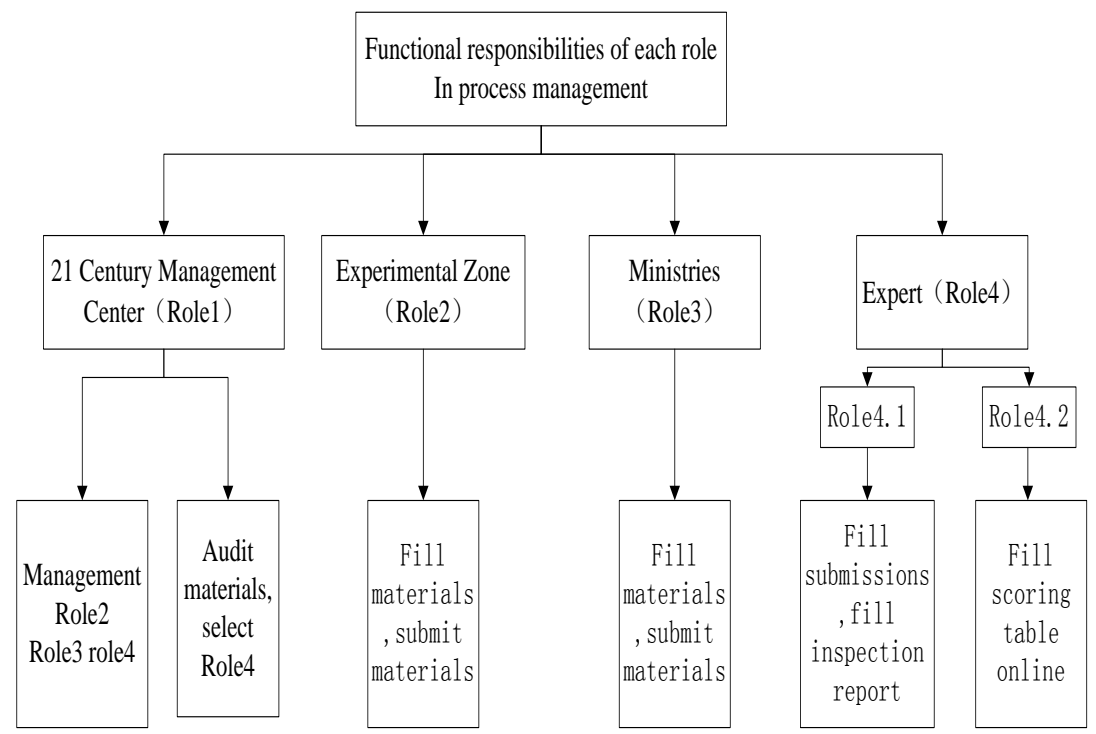

(a) Function responsibilities of roles

The function responsibilities of the roles which throughout declaration management, implement management and acceptance management in process management workflow. Declaration management is the start end of the workflow, implement management is center process and acceptance management is the end in the system.

Due to various roles will continue to change the status in the process of process management, so the system needs a mechanism which has the function of notice and adjust status, to perform real -time transmit and receive0 process management information, and transform various roles in different process management many process instances in which the state functions and adjust the corresponding operation. Thus, in the system a "state engine" module is designed to complete the above functions. After one role has completed a operation of a management process, "state engine" of the status operate sub-module to modify the role's state which corresponding to it; Receive the different business roles' "state engine" of real-time communication module information, " state engine" adjust roles' current state and refer the role to do what it is time to do according to the messages it received. The system uses a workflow state engine which operate and control process in Figure (b). 


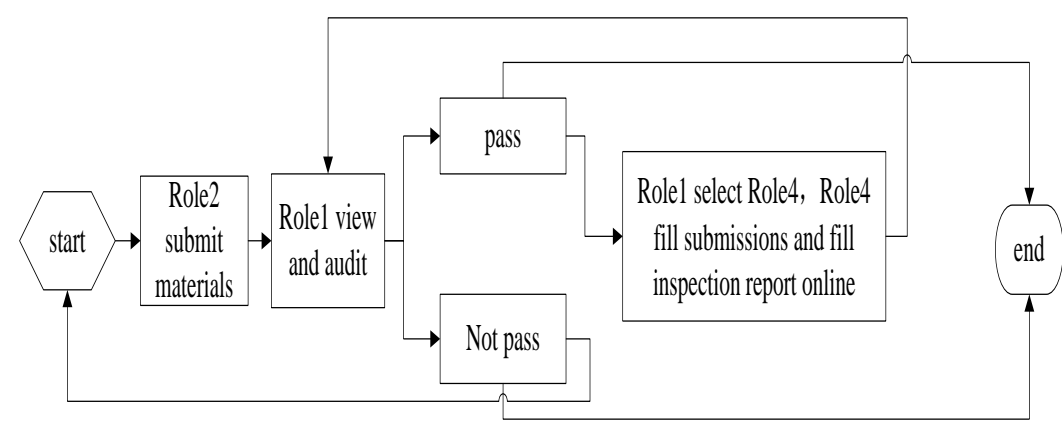

(b) workflow state engine

Workflow "state engine" automatically change status code in real-time according to the process workflow, and real-time communicate module refer various roles to do the current business. The work process of the system is as follows: first role2 submit materials, and then role1 will receive the notice of "state engine " which is refer it to audit the materials, if audit passed, "state engine" will notice role1 to select role4 , after the role4's instances selected ,they have the permissions to fill submissions and fill scoring table online. But if audition is not passed, "state engine" will be automatically initialized to the start state. When role4 has completed to fill submissions and scoring, the workflow engine will notice role1 status review, review by the declaration of the successful conclusion of the experimental area, else the failure to declare the end.

\section{The key technologies of process management module}

Database technology is a common method to implement workflow, and also the basis for the realization business workflow operating system. We use mysql database on Ecilspe integrated development environment to achieve a system of workflow business processes, the system uses a database connection by hibernate technology, its main work includes the following aspects:

JSP technology makes web developers and designers to develop Webpage rapidly and maintenance of dynamic Web page easily[6]. Web application can cross platform by using Jsp, which can run under Linux, can also run on other operating system. JSP has 2 kinds of structure, the system uses the Model2 MVC 
structure as shown in figure (c). MVC is the model, view, control the abbreviation, that it is to say that when using JSP, the corresponding file to realize the corresponding operation usually only responsible for displaying the page jsp. The business logic by Sevlet Action to realize.

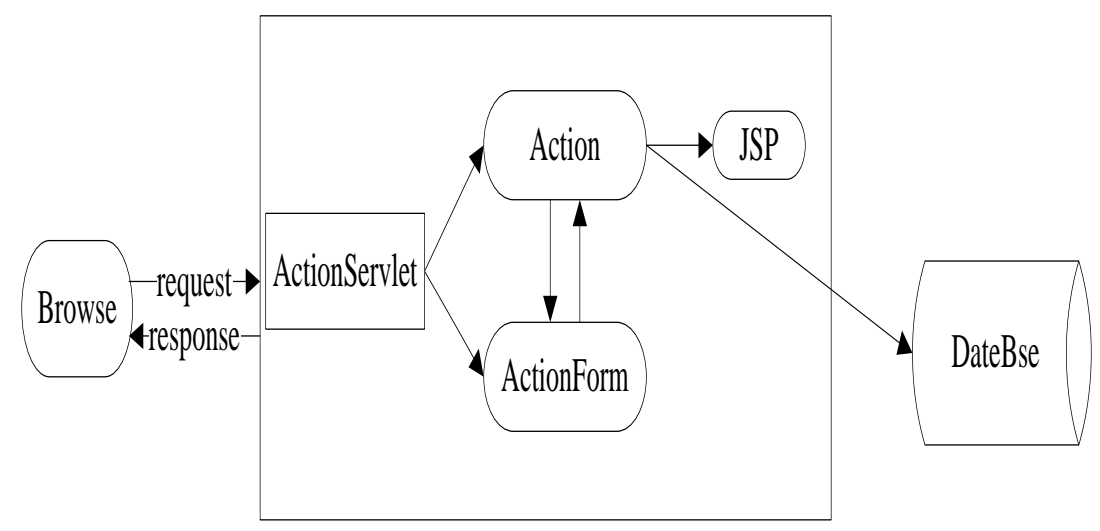

(c) MVC struts

Struts which is based on a model (Model) - View - controller (View) (Controller) (MVC) is open source framework for application architecture. MVC reduced the coupling between the business logic and data interface. Struts is a set of cooperating classes, Servlet and JSP markers, they form a reusable Model2 design. Struts taglib was used to obtain reusable code and abstract Java code, using the Action Servlet with Struts-config.xml to achieve the whole system navigation.

Hibernate[7] is an open source object-relational mapping framework , it has a very lightweight JDBC object package, the developer isolated from the database programming, Java programs operate all databases are handed out to deal with Hibernate it will automatically generate SQL statements to operate the database .Hibernate not only responsible for our mapping from Java entity to database tables (also including from Java data types to SQL data type mapping), also provides an object-oriented data query retrieval mechanism, thereby dramatically shortened development time manually processing SQL and JDBC on.

This system uses C3PO as a database connection pool, hibernate is 
configured to use $\mathrm{C} 3 \mathrm{PO}$ as connection pooling. C3PO includes a management class DBConnection-Manager, which is responsible for providing an interface between multiple connection pool object (DBConnectionPool class) with. Each connection pool object manages a set of JDBC Connection objects, each connection can be shared by any number of objects in the Servlet Action. С3PO class provides the following features:

(a) to obtain (or create) available connection from the connection pool.

(b) put connection back to the connection pool.

(c) release all resources and turn off all connections when the system is shut down

The system which uses C3PO connection pooling connection management strategy can effectively avoid the casual and no rules to use for the connection, you can make the connection multiplexing. By establishing a database connection pool, and a connection management strategy, making a database connection can be efficient and safe reuse, avoid frequent database connection is established, close overhead.

\section{Conclusion}

Based workflow management technology for sustainable urban development organization and management system, which can integrate many items of work, process management handled through Intranet. JSP technology uses cross-platform development, has a modular design, implementation, workflow specification, effectively carry out, using Mysql database as backend support. System with high efficiency, easy operation, convenient, safety, reliability, systems management in the 21st century and the experimental area has been effectively utilized.

\section{Acknowledgement}

In this paper, the research was sponsored by the National Technology R\&D P 
rogram(2013BAJ04B04).

\section{References}

[1] Yang Yue \& Zhang Yi, Analysis on Workflow Management System Based on UML Case Diagram Using [J]. Value Engineering, 2010,10:201-201.

[2] Li Xiang-Ning, Hao Ke-Gang \& ZhaoKe, A new Budiness Process Management Model[J]. Chinese journal of computers, Vol. 31 No. 1 Jan. 2008

[3] Rao Zhimin, Yu Yang \& Li Changshen. Research and Design of Workflow Client Application[J]. Application Research of Computers, 2007,4:212-213.

[4] Wang Jiachang, Xu Chang, Ji Xiaoming \& Ji Yong, On college students's tuition management system based on workflow[J].Computer application and software, 2012,6:294-297.

[5] Liu Xiaodong, Wang Beihu \& Kerridge , Achieving seam less componet composition through scenario based deep adaptation and generation. Science of Computer Programming 2005,56:157-170.

[6] Xie Xinjun \& He Zhijun, Design and Implementation of A Unique Form Workflow System.Computer Engineering,Vol.24,No. 9, September 1998.

[7] Yang Bin, Hao Ke_gang. The Solving Schemes of Workflow Management System based on Web[J]. Journal of Northwest University (Philosophy and Social Sciences Edition), 2009, 12:491-494.(in Chinese) 